\title{
Study of Different Adaptive Filter Algorithms for Noise Cancellation in Real-Time Environment
}

\author{
G.V.P.Chandra Sekhar Yadav \\ Student, M.Tech, DECS \\ Gudlavalleru Engineering College \\ Gudlavalleru-521356, Krishna District \\ Andhra Pradesh, India
}

\author{
B. Ananda Krishna, Ph.D \\ Professor, Department of ECE \\ Gudlavalleru Engineering College \\ Gudlavalleru-521356, Krishna District \\ Andhra Pradesh, India
}

\begin{abstract}
Adaptive filters are used in the situation where the filter coefficients have to be changed simultaneously according to the requirement. Adaptive filters are needed for fast convergence rate and low mean square error. Many algorithms have been proposed and proved that they have better convergence speed and tracking abilities. This paper shows the ability of adaptive filter for noise cancellation i.e., estimating the desired speech corrupted by unwanted signal i.e., noise. This paper is going to compare the performance of adaptive algorithms for noise cancellation in real time signals like recorded speech with different background noise. In the existing papers the authors have taken the input signal as sinusoidal signal etc. In order to measure the performance step size is the main factor for the convergence speed and mean square error. Many analyses proved that RLS algorithm had faster convergence speed and smaller steady state error compared with basic LMS algorithm and normalized LMS algorithm (NLMS). The existing simulation results enable to measure the performance of filter and show the convergence speed improvement when using RLS algorithm, NLMS algorithm and LMS algorithm.
\end{abstract}

\section{Keywords}

Adaptive filters; Wiener filters; Performance comparison; Convergence speed; Mean square error; Least mean square; Normalized least mean square; Recursive least square;

\section{INTRODUCTION}

Adaptive filters are variable filters whose filter coefficients are adjustable or modifiable automatically to improve its performance in accordance with some criterion, allowing the filter to adapt the changes in the input signal characteristics. Because of their self-adjusting performance and in-built flexibility, adaptive filters have found use in many diverse applications such as telephone echo cancelling, radar signal processing, and noise cancelling and biomedical signal enhancement. In any communication systems noise is the unwanted signal that mixes up with the desired signal; such noise is removed by using different techniques. In signal processing adaptive filters are the alternate method for recovering desired speech from the noise. Several algorithms have been proposed in earlier days to detect the desired signal. Least mean square (LMS) algorithm was the most efficient method in terms of computation and storage requirements but it has low convergence speed. After that normalized least mean square (NLMS) algorithm was proposed with moderate convergence speed but it was slow for colored input signals. Recursive least squares (RLS) algorithm has high convergence speed and tracking ability but this benefit comes under high computational cost. The paper consists of objective, existing work, proposed work, different parameters to be compared, filters, need for adaptive filters, adaptive algorithms, real time signals, existing simulation results, conclusion and future scope.

\section{OBJECTIVE}

The main objective of this project is to reduce the real time background noise that is inherently present in the signal and compare the performance of different algorithms in real time environment.

\section{EXISTING WORK}

The authors Yen-Hsiang Chen et al [2] proposed, "An Automotive Application of Real-time Adaptive Wiener Filter for Non-Stationary Noise Cancellation in a Car Environment".

In this paper, a real-time adaptive wiener filter with two micro phones is implemented to reduce noisy speech when noise signals and desired speech are incoming simultaneously. This paper also gives an analysis of different matrix sizes of the wiener filter so as to enable the possibility of real-time implementation. The performance of the proposed design is measured by as much as $20 \mathrm{~dB}$ noise reduction, and the proposed adaptive wiener matrix update speed achieves a 28.6 $\mathrm{ms} /$ frame, with a matrix size of 200 .

The authors H.kaur et al [3] proposed, "Performance and Convergence Analysis of LMS Algorithm".

In this paper, Instead of computing $W_{\text {opt }}$ as suggested by Wiener-Hopf equation, in LMS the coefficients are adjusted from sample to sample in such a way as to minimize the MSE. The LMS algorithm is based on steepest descent algorithm. This paper also gives the analysis of LMS algorithm for different input samples and different number of iterations. The simplicity of the LMS algorithm and ease of implementation make it first choice in many applications. The convergence rate is low for this algorithm.

The authors H.chang et al [4] proposed "A Variable Step size NLMS algorithm and its performance analysis".

In this paper, the step size of NLMS algorithm is varied according to the energy of the input signal.

NLMS algorithm is used to overcome the variable step size problem of LMS algorithm. The Normalized least mean squares filter (NLMS) is a variant of the LMS algorithm that solves variable step size problem by normalizing with the power of the input. The algorithm has moderate convergence rate.

The authors Talwar.R et al [1] proposed "performance comparison of adaptive algorithms for noise cancellation".

In this paper the authors have compared the performance of different adaptive algorithms like LMS, NLMS, and RLS 
algorithms for sinusoidal input. Performance is compared for different step size and different number of iterations. Three adaptive filter algorithms have been compared by simulation to achieve high convergence rate and minimum mean square error with noise and different values of $\mu$. Every algorithm works on different methods for noise cancellation and improves system performance.

\section{PROPOSED WORK}

In the existing work, the authors H.Kaur and R.Talwar [1] compared the performance of adaptive filter algorithms like LMS, NLMS and RLS algorithms for sinusoidal input signals. Now, the same work is going to extend with the real-time signal like recorded speech and compare the performance of adaptive algorithms LMS, NLMS and RLS algorithms for different step size and different number of iterations. The performance of the algorithms is going to analyze with different input speech signals with different lengths and parameters.

\section{PARAMETERS}

Performance of the filters is measured with different parameters like convergence rate, mean square error, tracking, mis-adjustment etc. Step size is the major factor for convergence speed. In filters number of coefficients is the main parameter that affects the performance of minimum mean square error (MMSE) of adaptive filter. MMSE is a monotonic non increasing function of tap length but due to increase of tap length, there is a decrease in MMSE. It doesn't increase complexity but introduces adaptation noise. So it is better to use an optimum tap length that balances the steady state performance and complexity. In echo cancelling system with multi input the echo length may be keep changing according to the requirement, but most of the cases tap length is fixed.

\section{FILTERS}

Filtering is used to process a signal in such a way that signal to noise ratio is enhanced. When the signal and noise are stationary and their characteristics are known or can be assumed, fixed filters are used. The design of fixed filters is based on prior knowledge of signal and noise characteristics. Adaptive filters can be defined as a filter that can automatically adjust their own parameters, based on the incoming signal and noise characteristics.

\subsubsection{Basic wiener filter theory}

Wiener filter is a statistical method where the estimation of signal is known before processing the signal [2]. Wiener theory defines the optimum value of the filter. The mean square error (MSE), $\xi$, is defined by the "expectation" of the squared error $\left(e_{k}\right)$.

Many adaptive algorithms can be viewed as approximations of the discrete wiener filter. Two signals $x_{k}$ and $y_{k}$ are applied to the filter. Typically $y_{k}$ consists of a component that is correlated with $x_{k}$ and another that is not. The wiener filter produces an optimal estimate of the part of $y_{k}$ that is then subtracted from $y_{k}$ to yield error $\left(e_{k}\right)$ as shown in fig 1 .

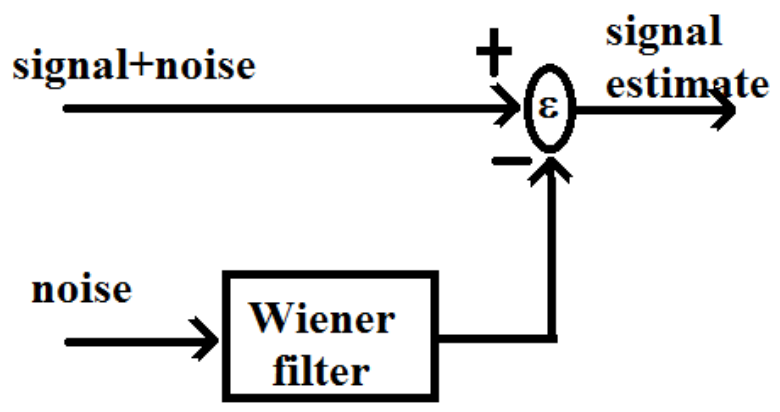

Fig 1: Wiener filter

$$
e_{k}=y_{k}-\widehat{n_{k}}=y_{k}-\sum_{i=0}^{N-1} w(i) \cdot x_{k-i}
$$

Where $\mathrm{x}_{\mathrm{k}}$ and $\mathrm{w}$ are the input signal vector and weight vector respectively are given by

$$
\begin{gathered}
x_{k}=\left[x_{k} x_{k-1} x_{k-2} x_{k-3} \ldots \ldots x_{k-(N-1)}\right]^{T} \\
w=[w(0) w(1) w(2) w(3) \ldots \ldots . w(N-1)]^{T}
\end{gathered}
$$

The mean square error (MSE) is obtained by taking expectation to square of the error $\mathrm{e}_{\mathrm{k}}^{2}$.

$$
\xi=\in\left\{e_{k}^{2}\right\}=\in\left\{y_{k}^{2}\right\}-2 W^{T} R_{Y X}+W^{T} R_{X X} W
$$

Weiner filter theory is defined by performance surface [6]-[7].

\subsubsection{Performance surface}

A plot of MSE against the filter coefficient $W$ is bowl shaped with a unique bottom is shown in figure 2 . It is known as the performance surface and is Non-negative. The gradient of the performance surface is given by

$$
\nabla=\frac{d \xi}{d w}=0-2 P+2 R W
$$

Where $P=R_{Y X}$ is the N-length cross correlation vector $R=R_{X X}$ is the $N \times N$ auto correlation matrix.

At minimum point of surface, the gradient is zero and filter weight vector has its optimum value $W_{\text {opt }}$.

$$
\begin{gathered}
\nabla=0 \\
-2 P+2 R W=0 \\
2 P=2 R W \\
W_{\text {opt }}=R^{-1} P
\end{gathered}
$$

This is known as the wiener-Hopf equation or solution [2] The task in adaptive filtering is to adjust the filter weights $w(0) w(1) w(2) w(3) \ldots \ldots$ using a suitable algorithm, to find the optimum point on the performance surface.

The wiener filter has a limited practical usefulness because of the following reasons.

It requires the auto correlation matrix $\mathrm{R}$ and the cross correlation vector $\mathrm{P}$ both of which are not known. It involves matrix inversion, which is time consuming.

If the signals are non-stationary then both $\mathrm{R}$ and $\mathrm{P}$ will change with time and so $W_{\text {opt }}$ will have to be computed repeatedly.

For real time applications, a way of obtaining $W_{\text {opt }}$ on a sample by sample basis is required. Adaptive algorithms are used to achieve this without having to compute $\mathrm{R}$ and $\mathrm{P}$ explicitly or performing a matrix inversion. The solution to Wiener-Hopf equation is the steepest descent algorithm [10]. 


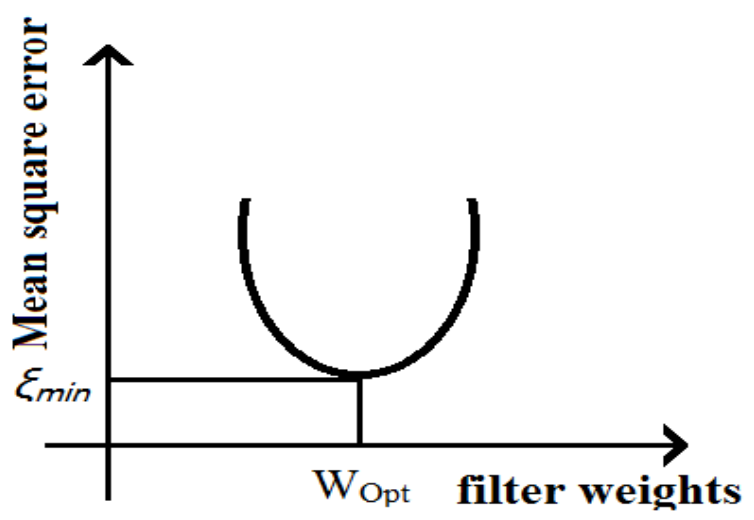

Fig 2: Error performance surface

\subsubsection{Adaptive filters}

Adaptive filter is essentially a digital filer with self-adjusting characteristics. It adapts automatically to changes in its input signals [6].

The contamination of a signal of interest by other unwanted signal or noise is a problem often encountered in many applications. Where the signal and noise occupy fixed and separate frequency bands, conventional linear filters with fixed coefficients are normally used to extract the signal. However, there are many instances when it is necessary for the filter characteristics to be variable, adapted to changing signal characteristics or to be altered intelligently.

In such cases, the coefficients of the filter must vary and can't be specified in advance. Such is the case where there is a spectral overlap between the signal and noise or if the band occupied by the noise is unknown or varies with time [7].

Adaptive filters are used in the following cases:-

When it is necessary for the filter characteristics to be variable, adapted to changing conditions

When there is spectral overlap between signal and noise

If the band occupied by the noise is unknown or varies with time

The use of conventional filters in the above cases would lead to unacceptable distortion of the desired signal.

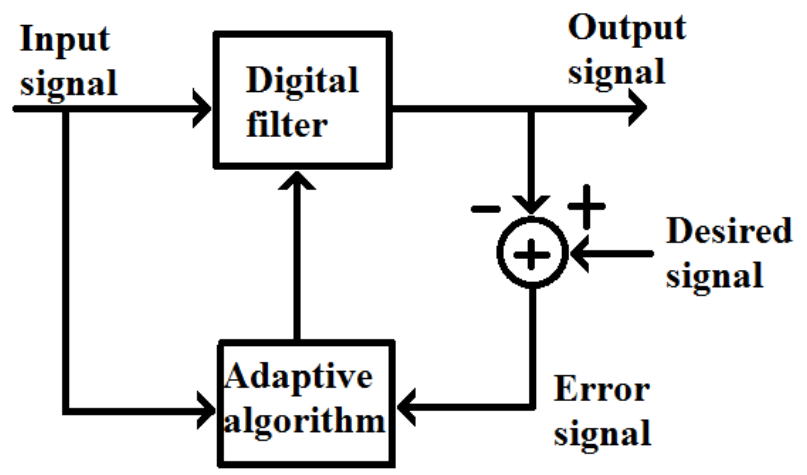

Fig 3: Adaptive filter

Fig 3 shows the basic adaptive filter with input signal and desired signal as inputs and one output signal with adaptive algorithm to adapt changes in input signal.

\subsubsection{Adaptive filter as noise canceller}

An adaptive filter consists of two distinct parts: a digital filter with adjustable coefficients, and an adaptive algorithm which is used to adjust or modify the coefficients of the filter.

Two input signals $y_{k}$ and $x_{k}$ are applied simultaneously to the adaptive filter. The signal $y_{k}$ is the contaminated signal containing both the desired signal $s_{k}$ and the noise $n_{k}$ assumed uncorrelated with each other. The signal $x_{k}$ is a measure of the contaminating signal which is correlated in same way with $n_{k}$.

$x_{k}$ is processed by the digital filter to produce an estimate, $\widehat{n_{k}}$ of $n_{k}$. An estimate of the desired signal is then obtained by subtracting the digital filter output, $\widehat{n_{k}}$ from the contaminated signal $y_{k}$

$$
\widehat{s_{k}}=y_{k}-\widehat{n_{k}}=s_{k}+n_{k}-\widehat{n_{k}}
$$

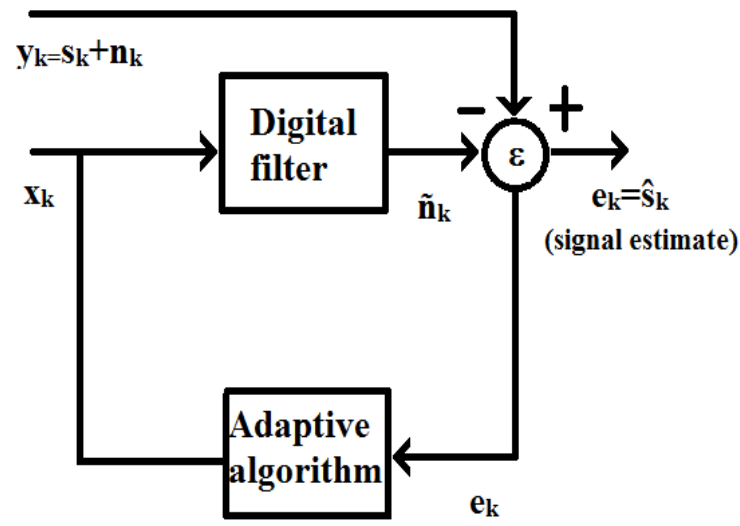

Fig 4: Block diagram of adaptive filter as noise canceller

The main objective in noise cancelling is to produce an optimum estimate of the noise in the contaminated signals and hence an optimum estimates of the desired signal [12].

This is achieved by using $\widehat{s_{k}}$ in a feedback arrangement to adjust the digital filer coefficients, via a suitable adaptive algorithm, to minimize the noise in $\widehat{s_{k}}$ as shown in fig 4 .

The output signal $\widehat{s_{k}}$ serves two purposes:

1) As an estimate of the desired signal and

2) As an error signal which is used to adjust the filter coefficients.

\section{ADAPTIVE ALGORITHMS}

Adaptive algorithms are used to adjust the coefficients of the digital filter. Such that the error signals $e_{k}$ is minimized according to some criterion.

The most commonly used adaptive algorithms are least mean square (LMS), normalized least mean square (NLMS) because of their robustness and simplicity and also have recursive least square (RLS) algorithm for noise cancellation.

\subsubsection{Least mean square (LMS) algorithm}

One of the most successful adaptive algorithms is the LMS algorithm. Instead of computing $W_{\text {opt }}$ in one go as suggested by wiener-Hopf equation, in the LMS the coefficients are adjusted from sample to sample in such or way as to minimize the MSE [3].

This amounts to descending along the surface of the figure 2 towards its bottom.

The LMS algorithm is based on the steepest descent algorithm where the weight vector is updated from sample to sample as follows

$$
W_{k+1}=W_{k}-\mu \nabla_{k}
$$


Where $W_{k}$ and $\nabla_{k}$ are the weight and the true gradient vectors respectively at the $k^{\text {th }}$ sampling instant. $\mu$ controls the stability and rate of convergence.

The steepest descent algorithm in the above equation still requires knowledge of $\mathrm{R}$ and $\mathrm{P}$. since $\nabla_{k}$ is obtained by evaluating the equation.

$$
\nabla=\frac{d \xi}{d w}=0-2 P+2 R W
$$

The LMS algorithm is a practical method of obtaining estimates of the filter weights $W_{k}$ in real time without matrix inversion in the equation $W_{\text {opt }}=R^{-1} P$ or the direct computation of the auto correlation and cross correlation.

$$
\nabla=-2 P+2 R W
$$

In the LMS algorithm, instantaneous estimates are used for $\nabla$. Thus

$e_{k}=y_{k}-x_{k}^{T} W_{k}$

$$
\begin{gathered}
\nabla_{k}=-2 P+2 R W \\
\nabla_{k}=-2 x_{k} y_{k}+2 x_{k} x_{k}^{T} W_{k} \\
\nabla_{k}=-2 x_{k}\left(y_{k}-x_{k}^{T} W_{k}\right) \\
\nabla_{k}=-2 x_{k} e_{k}
\end{gathered}
$$

replace the value of $\nabla_{k}$ in steepest descent algorithm yields

$$
\begin{gathered}
W_{k+1}=W_{k}-\mu \nabla_{k} \\
W_{k+1}=W_{k}-\mu\left(-2 x_{k} e_{k}\right) \\
W_{k+1}=W_{k}+2 \mu e_{k} x_{k}
\end{gathered}
$$

Clearly, the LMS algorithm above doesn't require prior knowledge of the signal statistics (that is the correlations $\mathrm{R}$ and $\mathrm{P}$ ), but instead uses their instantaneous estimates as shown above.

The weights obtained by the LMS algorithm are only estimates, but these estimates improve gradually with time as weights are adjusted and the filter learns the characteristics of the signals. Eventually, the weights converge [9].

The condition of convergence is

$$
0<\mu>1 / \lambda_{\operatorname{Max}}
$$

Where $\lambda_{\text {Max }}$ is the maximum Eigen value of the input data covariance matrix.

In general $\mathrm{W}_{\mathrm{k}}$ never reaches the theoretical optimum (the wiener solution), but fluctuates about it as shown in fig5.

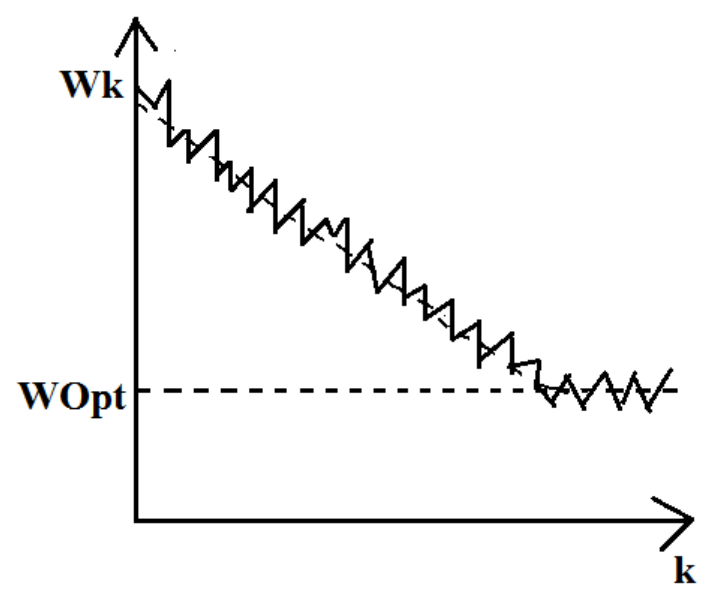

Fig 5: An illustration of the variations in the filter weights

The simplicity of the LMS algorithm and ease of implementation, make it the algorithm of first choice in many real-time systems [11]. The LMS algorithm requires approximately $2 \mathrm{~N}+1$ multiplications and $2 \mathrm{~N}+1$ additions for each new set of input and output samples [3].

Most signal processors are suited to the mainly multiply accumulate arithmetic operations involved, making a direct implementation of the LMS algorithm attractive.

\subsubsection{Normalized least mean square (NLMS) algorithm}

The main drawback of the pure LMS algorithm is that it is sensitive to the scaling of its input $x_{k}$. This makes it very hard to choose a learning rate $\mu$ that guarantees stability of the algorithm [5].

The normalized least mean square filter is a variant of LMS algorithm that solves this problem by normalizing with the power of input.

Where $\mu_{k}=\frac{\mu}{x_{k+1}^{T} x_{k}} ; 0<W_{k}+\mu_{k} x_{k} e_{k}$
$0<2$

In the previous equation, the NLMS algorithm becomes the same as the standard LMS algorithm except that the NLMS algorithm has a time varying step $\operatorname{size} \mu_{k}$. The step size improves the convergence speed of adaptive filter [8].

In practice, at some time $x_{k}$ can be very small. To make NLMS algorithm more robust, it can be modified as

$$
W_{k+1}=W_{k}+\frac{\mu}{\delta+x_{k}^{T} x_{k}} x_{k} e_{k}
$$

So, that the gain constant can't go to infinity.

\subsubsection{Recursive least squares (RLS) algorithm}

The Recursive least squares (RLS) adaptive filter is an algorithm which recursively finds the filter coefficients that minimize a weighted linear least squares cost function relating to the input signals. This is in contrast to other algorithms such as the least mean squares (LMS) that aim to reduce the mean square error. In the derivation of the RLS, the input signals are considered deterministic, while for the LMS and similar algorithm they are considered stochastic. Compared to most of its competitors, the RLS exhibits extremely fast convergence. However, this benefit comes at the cost of high computational complexity.

The idea behind RLS filters is to minimize a cost function C by appropriately selecting the filter coefficients $W_{k}$, updating the filter as new data arrives. The error signals $e_{k}$ and desired signal $y_{k}$ is defined in the negative feedback.

The error implicitly depends on the filter coefficients through the estimate $\widehat{n_{k}}$

$$
e_{k}=y_{k}-\widehat{n_{k}}
$$

The weighted least squares error function $\mathrm{C}$ - function has to minimize being a function of $e_{k}$ is therefore also dependent on the filter coefficients.

$$
C\left(W_{k}\right)=\sum_{i=0}^{k} \lambda^{n-i} e_{k}^{2}
$$

Where $0<\lambda \leq 1$ is the "forgetting factor" which gives exponentially less weight to older error samples.

The cost function is minimized by taking the partial derivatives for all entries of the coefficient vector $W_{k}$ and setting the results to zero.

Finally the RLS algorithm for an $p^{\text {th }}$ order filter can be

$$
\begin{gathered}
\alpha_{k}=y_{k}-x_{k}^{T} W_{k-1} \\
g_{k}=p_{k-1} x_{k}^{*}\left\{\lambda+x_{k}^{T} p_{k-1} x_{k}^{*}\right\}^{-1}
\end{gathered}
$$




$$
\begin{gathered}
p_{k}=\lambda^{-1} p_{k-1}-g_{k} x_{k}^{T} \lambda^{-1} p_{k-1} \\
W_{k}=W_{k-1}+\alpha_{k} g_{k}
\end{gathered}
$$

Where $\mathrm{p}=$ filter order

$\lambda=$ forgetting factor

$\delta=$ value to initialize $p_{0}$

$W_{k}=0$

$p_{0}=\delta^{-1} I$ Where I is the identity matrix of rank $\mathrm{p}+1$

\section{REAL TIME SIGNALS}

All these algorithms discussed above take one of the inputs as any random signal and mixes with random noise and these algorithms eliminates the random noise from the signal. Now, here these input random signals are replaced with the realtime signals like recorded speech with different background noises and the algorithms are used to remove that background noise that is inherently present in the recorded speech.

In mat lab speech is recorded by using the instruction wavrecord: record sound using PC-based audio input device.

Syntax is

Wavrecord (n, fs, 'dtype');

Where $\mathrm{n}$ indicates number of samples of the audio signal to be recorded

fs indicates sampling rate, the default value is $11025 \mathrm{~Hz}$

dtype used to record sound, the string dtype can be one of the following

'double' (default value), 16 bits/sample

'single', 16 bits/sample

'int16', 16 bits/sample

'uint8', 8 bits/sample

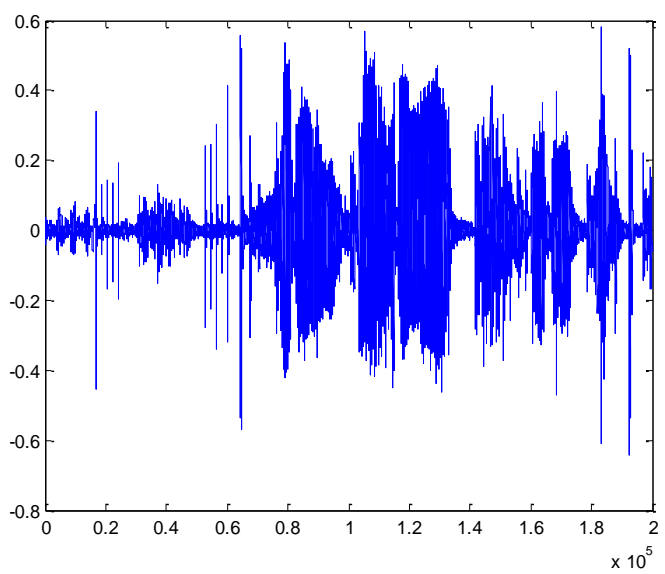

Fig 6:Real-time speech signal

The recorded speech as shown in fig 6 is mixes with different background noises and these background noises are removed by using different adaptive algorithms.

\section{EXISTING SIMULATION RESULTS}

The result analysis of LMS, NLMS and RLS algorithms proves that RLS algorithm provides efficient convergence rate than LMS, NLMS algorithms. Figure 7 shows the comparison between three algorithms for noise cancellation with sinusoidal signal as the input signal. From the figure 7 it is clear that NLMS algorithm provides better results from the other two methods i.e., wiener and LMS algorithm. Wiener filter has to compute R and P explicitly but LMS algorithm is suited for real time applications where sample by sample bias is done.

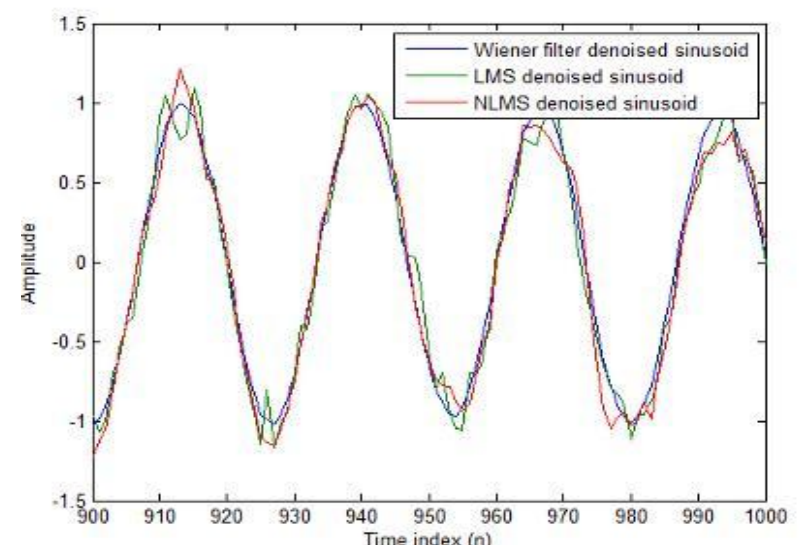

Fig 7: Comparison between wiener, LMS and NLMS for output

Figure 8 shows the comparison between LMS and NLMS for mean square error. NLMS algorithm provides the solution for variable step size problem and removes noise better than LMS algorithm. MSE is used to analyze the performance by minimizing it.

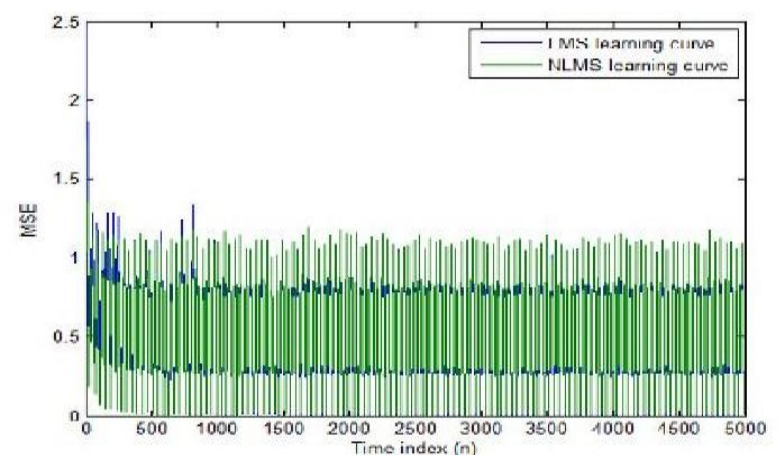

Fig 8: Comparison between LMS and NLMS algorithm for MSE

\section{CONCLUSION AND FUTURE SCOPE}

Several authors proposed different algorithms for noise cancellation and compared their performance for different step size values. Out of which RLS algorithm provides better results than other algorithms but has high degree of complexity. The computational complexity and stability problem increases in an algorithm as the authors tried to reduce the mean squared error. NLMS is the favorable choice for most of the industries due less computational complexity and fair amount of noise reduction. All of these algorithms are worked on different parameters to remove noise but these algorithms use input signals as sinusoidal with random noise signal. As a future work, the same work is going to extend in real time environment like recorded speech with different background noise and compare their performance with different algorithms i.e., LMS, NLMS and RLS to achieve high convergence rate. The performance is analyzed with different parameters and lengths of input signal.

\section{REFERENCES}

[1] Kaur. H and Talwar. R, "Performance comparison of adaptive algorithms for noise cancellation", Engineering trends in communication, C2SPCA 2013.

[2] Yen-Hsiang chen, Shanq-Jang Ruan, Tom Qi, "An Automotive Application of real time adaptive wiener 
filter for noise cancellation in a car environment," IEEE,2012,4673-2193.

[3] H.Kaur and R.Talwar, "Performance and Convergence Analysis of LMS Algorithm,” IEEE ICCIC, Dec.2012.

[4] H.Chang and J.Lee, "A Variable Step Size NLMS Algorithm and its Performance Analysis," IEEE Trans. Signal Process, vol. 60, no. 4, April 2012.

[5] H.Chang and J.Lee, "Performance Comparison of Variable Step Size NLMS Algorithms," World Congress on Eng. And Computer Science, vol. 1, no. 4, Oct. 2009.

[6] B.Widrow and S.D.Stearns, Adaptive Signal Processing, Englewood Cliffs, NJ: Prentice- Hall, 1985.

[7] S .Haykin, Adaptive Filter Theory, Fourth edition, Upper saddle River, NJ: Prentice -Hall, 2002.
[8] C. Paleologu, J Benesty and S.LChristopher "Variable Step Size NLMS Algorithm designed for Echo Cancellations," IEEE 2009.

[9] J.Gorriz and J.Ramrez, "A Novel LMS Algorithm Applied to Adaptive Noise Cancellation," IEEE Signal Process Letters, vol. 16, no. 1, Jan. 2009.

[10] K. A. Lee, W. S. Gan, and S. M. Kuo, Subband Adaptive Filtering: Theory and Implementation. Hoboken, NJ: Wiley, 2009.

[11] C.Gabriela and M.Sarachin, "Echo Cancellation Using LMS Algorithm," U.P.B Sci Bull., Series C, vol. 71, no. 4, 2009.

[12] B.Widrow, J.R.Glover "Adaptive Noise Cancelling: Principles and Applications," IEEE Proceedings, Vol-63, No.12, Dec. 1975. 